\title{
Description of Turbulent Events Through the Analysis of POD Modes in Numerically Simulated Turbulent Channel Flow
}

\author{
Giancarlo Alfonsi ${ }^{1}$ and Leonardo Primavera ${ }^{2}$ \\ ${ }^{1}$ Dipartimento di Difesa del Suolo, Università della Calabria , \\ Via P. Bucci 42b, 87036 Rende (Cosenza), Italy \\ alfonsi@dds. unical.it \\ ${ }^{2}$ Dipartimento di Fisica, Università della Calabria, \\ Via P. Bucci 33b, 87036 Rende (Cosenza), Italy \\ lprimaveralfis.unical.it
}

\begin{abstract}
The flow of a viscous incompressible fluid in a plane channel is simulated numerically with the use of a parallel computational code for the numerical integration of the Navier-Stokes equations. The numerical method is based on a mixed spectral-finite difference algorithm and the approach of the Direct Numerical Simulation (DNS) is followed in the calculations. A turbulent-flow database is constructed, including 500 non-dimensional time steps of the turbulent statistically steady state flow field at Reynolds number $R e_{\tau}=180$. The coherent structures of turbulence are extracted from the fluctuating portion of the velocity field with the use of the Proper Orthogonal Decomposition technique (POD). Turbulent events occurring in the flow are described in terms of temporal dynamics of the coherent turbulent structures, the flow modes educed with the POD technique.
\end{abstract}

\section{Introduction}

The properties of turbulence in wall bounded flows have been investigated with the use of a variety of techniques and methods. Accurate and extensive works in which a considerable amount of results have been reviewed are due to Robinson [1] and Panton [2]. Vortical structures of various kind have been observed in the inner region of wall-bounded flows, following different mechanisms of self-sustainment (Panton [2]). A fist type of mechanism involves the generation of a streamwise vortex from an already existing streamwise vortex, near either end of the original vortex. A second type of mechanism involves the generation of an horseshoe vortex from a parent horseshoe vortex and the related interaction of the vortex legs, that includes the lift-up of the vortex head. A third mechanism is the bridging between two streamwise fingers of vorticity. Other phenomena occur in the outer region, like the regeneration of hairpin vortices into packets with the vortical structures of the outer region interacting with those of the inner region.

In spite of the large amount of scientific work accomplished, still there are no definite conclusions on the character of the phenomena occurring in the near-wall 
region of a wall-bounded turbulent flow. This is due to the complexity of turbulence itself and to the quality of the scientific information that is possible to gather with the use of the current research techniques.

Contemporary times are characterized by the fact that high-performance computers are frequently used in turbulence research. In computational fluid dynamics a relevant issue of is that of the method that has to be followed to take into account the phenomenon of turbulence in the numerical simulations. The most rigorous approach is that of the Direct Numerical Simulation (DNS) according to which the objective of calculating all turbulent scales is pursued and the Navier-Stokes equations are numerically integrated without modifications. The crucial aspect of this method is the accuracy of the calculations that in theory should to be sufficiently high as to resolve the Kolmogorov microscales in space and time. DNS results for the case of the plane channel have been reported, among others, by Kim et al. [3] and Moser et al. [4].

Modern techniques for the numerical integration of the Navier-Stokes equations in conjunction with the increasing power of computers have the ability of greatly increasing the amount of data gathered during a research of computational nature. Moreover, the effort of studying turbulence in its full complexity has brought to the condition of managing large amounts of data. A typical turbulent-flow database includes all three components of the fluid velocity in all points of a three-dimensional domain, gathered for an adequate number of time steps of the turbulent statistically steady state. Such a database contains a considerable amount of information about the physics of the flow and, in the formation of the instantaneous value of each variable, all turbulent scales have contributed, being the effect of each scale nonlinearly combined with all others. Methods can be applied in order to extract from a turbulentflow database only the relevant information, by separating the effects of appropriately-defined modes of the flow from the background flow, i.e. extract the coherent structures of turbulence.

In this work the issue of the coherent structures of turbulence in the wall region of a turbulent channel flow is addressed. The coherent turbulent motions are educed with the technique of the Proper Orthogonal Decomposition (POD) from a numerical database that has been built with the use of a parallel, three-dimensional, timedependent computational code for the numerical integration of the Navier-Stokes equations. The DNS approach has been followed in the calculations.

\section{Numerical Techniques}

The numerical simulations have been performed with a parallel computational code based on a mixed spectral-finite difference algorithm. The system of the unsteady Navier-Stokes equations for incompressible fluids in three dimensions and nondimensional conservative form is considered $(i \& j=1,2,3)$ :

$$
\begin{gathered}
\frac{\partial u_{i}}{\partial x_{i}}=0 \\
\frac{\partial u_{i}}{\partial t}+\frac{\partial}{\partial x_{j}}\left(u_{i} u_{j}\right)=-\frac{\partial p}{\partial x_{i}}+\frac{1}{R e_{\tau}} \frac{\partial^{2} u_{i}}{\partial x_{j} \partial x_{j}}
\end{gathered}
$$


where $u_{i}(u, v, w)$ are the velocity components in the cartesian coordinate system $x_{i}(x, y, z)$. Equations (1) are nondimensionalized by the channel half-width $\delta$ for lenghts, wall shear velocity $u_{\tau}=\sqrt{\tau_{w} / \rho}$ for velocities, $\rho u_{\tau}^{2}$ for pressure and $\delta / u_{\tau}$ for time, being $R e_{\tau}=\left(u_{\tau} \delta / v\right)$ the friction Reynolds number. The fields are admitted to be periodic in the streamwise $(x)$ and spanwise $(z)$ directions, and equations (1) are Fourier transformed accordingly. The nonlinear terms in the momentum equation are evaluated pseudospectrally by anti-transforming the velocities back in physical space to perform the products (FFTs are used). A dealiasing procedure is applied to avoid errors in transforming the results back to Fourier space. In order to have a better spatial resolution near the walls, a grid-stretching law of hyperbolic-tangent type has been introduced for the grid points along $y$, the direction orthogonal to the walls. For the time advancement, a third-order Runge-Kutta algorithm has been implemented and the time marching procedure is accomplished with the fractional-step method (Kim et al. [3]). No-slip boundary conditions at the walls and cyclic conditions in the streamwise and spanwise directions have been applied to the velocity. More detailed descriptions of the numerical scheme, of its reliability and of the performance obtained on the parallel computers that have been used, can be found in Alfonsi et al. [5] and Passoni et al. [6],[7],[8].

\section{Turbulent-Flow Database}

By recalling the wall formalism, one has: $x_{i}^{+}=x_{i} u_{\tau} / \nu=x_{i} / \delta_{\tau}, t^{+}=t u_{\tau}^{2} / \nu=t u_{\tau} / \delta_{\tau}$, $\delta^{+}=\delta / \delta_{\tau}, u^{+}=\bar{u} / u_{\tau}, R e_{\tau}=u_{\tau} \delta / v=\delta / \delta_{\tau}=\delta^{+}$, where $\bar{u}$ is streamwise velocity averaged on a $x-z$ plane and time, $\delta_{\tau}=v / u_{\tau}$ is the viscous length and $\delta / u_{\tau}$ the viscous time unit. In Table 1 the characteristic parameters of the numerical simulations are reported.

Table 1. Characteristic parameters of the numerical simulations

\begin{tabular}{|c|c|c|c|c|c|c|c|c|}
\hline \multicolumn{3}{|c|}{ Computing domain } & \multicolumn{3}{|c|}{ Computational grid } & \multicolumn{3}{|c|}{ Grid spacing } \\
\hline$L_{x}$ & $L_{y}$ & $L_{z}$ & $N_{x}$ & $N_{y}$ & $N_{z}$ & $\Delta x^{+}$ & $\Delta y_{\text {wall }}^{+}$ & $\Delta z^{+}$ \\
\hline $2 \pi \delta$ & $2 \delta$ & $\pi \delta$ & 96 & 129 & 64 & 11.8 & 0.87 & 8.8 \\
\hline
\end{tabular}

It can be verified that in the present work there are 8 grid points in the $y$ direction, within the viscous sublayer $\left(y^{+} \leq 7\right)$. The Kolmogorov spatial microscale, estimated using the criterion of the average dissipation rate per unit mass across the width of the channel, results $\eta^{+} \approx 1.8$. After the insertion of appropriate initial conditions, the initial transient of the flow in the channel has been first simulated, the turbulent 
statistically steady state has been reached and then calculated for a time $t=10 \delta / u_{\tau}$ $\left(t^{+}=1800\right)$ to form a 500 time-step database with a temporal resolution of $\Delta t=2 \times 10^{-2} \delta / u_{\tau}\left(\Delta t^{+}=3.6\right)$ between each instant. In Table 2 predicted and computed values of a number of mean-flow variables are reported.

Table 2. Predicted vs. computed mean-flow variables

Predicted variables

\begin{tabular}{ccccccl}
\hline$R e_{\tau}$ & $R e_{b}$ & $R e_{c}$ & $U_{b} / u_{\tau}$ & $U_{c} / u_{\tau}$ & $U_{c} / U_{b}$ & $C_{f b}$ \\
180 & 2800 & 3244 & 15.56 & 18.02 & 1.16 & $8.44 \times 10^{-3}$
\end{tabular}

Computed variables

\begin{tabular}{lcrlccl}
\hline$R e_{\tau}$ & $R e_{b}$ & $R e_{c}$ & $U_{b} / u_{\tau}$ & $U_{c} / u_{\tau}$ & $U_{c} / U_{b}$ & $C_{f b}$ \\
178.74 & 2786 & 3238 & 15.48 & 17.99 & 1.16 & $8.23 \times 10^{-3}$ \\
\hline
\end{tabular}

In Table 2, $U_{b}$ and $U_{c}$ are the bulk mean velocity and the mean centerline velocity respectively, while $R e_{b}$ and $R e_{c}$ are the related Reynolds numbers. The predicted values of $U_{c} / U_{b}$ and $C_{f b}$ are obtained from the correlations suggested by Dean [9]:

$$
\frac{U_{c}}{U_{b}}=1.28\left(2 R e_{b}\right)^{-0.0116} ; \quad C_{f b}=0.073\left(2 R e_{b}\right)^{-0.25} .
$$

The computed skin friction coefficient is defined as:

$$
C_{f b}=\frac{\tau_{w}}{\frac{1}{2} \rho U_{b}^{2}}
$$

and is evaluated from the actual shear stress at the wall obtained in the computations. procedure is followed for the evaluation of the friction velocity in the computed $R e_{\tau}$.

\section{Proper Orthogonal Decomposition}

The Proper Orthogonal Decomposition is a technique that can be applied for the extraction of the coherent structures from a turbulent flow field (Berkooz et al. [10], Sirovich [11]). By considering an ensemble of temporal realizations of a velocity field $u_{i}\left(x_{j}, t\right)$ on a finite domain $D$, one wants to find which is the most similar function to the elements of the ensemble, on average. This problem corresponds to find a deterministic vector function $\varphi_{i}\left(x_{j}\right)$ such that $(i \& j=1,2,3)$ : 


$$
\max _{\psi} \frac{\left\langle\left|\left(u_{i}\left(x_{j}, t\right), \psi_{i}\left(x_{j}\right)\right)\right|^{2}\right\rangle}{\left(\psi_{i}\left(x_{j}\right), \psi_{i}\left(x_{j}\right)\right)}=\frac{\left\langle\left|\left(u_{i}\left(x_{j}, t\right), \varphi_{i}\left(x_{j}\right)\right)\right|^{2}\right\rangle}{\left(\varphi_{i}\left(x_{j}\right), \varphi_{i}\left(x_{j}\right)\right)} .
$$

A necessary condition for problem (6) is that $\varphi_{i}\left(x_{j}\right)$ is an eigenfunction, solution of the eigenvalue problem and Fredholm integral equation of the first kind:

$$
\int_{D} R_{i j}\left(x_{l}, x_{l}^{\prime}\right) \varphi_{j}\left(x_{l}^{\prime}\right) d x_{l}^{\prime}=\int_{D}\left\langle u_{i}\left(x_{k}, t\right) u_{j}\left(x_{k}^{\prime}, t\right)\right\rangle \varphi_{j}\left(x_{k}^{\prime}\right) d x_{k}^{\prime}=\lambda \varphi_{i}\left(x_{k}\right)
$$

where $R_{i j}=\left\langle u_{i}\left(x_{k}, t\right) u_{j}\left(x_{k}^{\prime}, t\right)\right\rangle$ is the two-point velocity correlation tensor. To each eigenfunction $\varphi_{i}^{(n)}\left(x_{j}\right)$ is associated a real positive eingenvalue $\lambda^{(n)}$ and every member of the ensemble can be reconstructed by means of a modal decomposition in the eigenfunctions themselves:

$$
u_{i}\left(x_{j}, t\right)=\sum_{n} a_{n}(t) \varphi_{i}^{(n)}\left(x_{j}\right)
$$

that can be seen as a decomposition of the originary random field into deterministic structures with random coefficients.

In the present work the POD technique is applied for the analysis of the fluctuating portion of the velocity field in a plane channel at $\operatorname{Re}_{\tau}=180$. Besides the timeaveraged quantities outlined above, the decomposition properties are used to compute two time-dependent quantities (Webber et al. [12]), the kinetic energy of the fluctuations $E(t)$ and the representational entropy $S(t)$, respectively:

$$
\begin{gathered}
E(t)=\int_{D} u_{i}(x, y, z, t) u_{i}(x, y, z, t) d x_{i}=\sum_{n} a^{n}(t) a^{-n}(t) \\
S(t)=-\sum_{n} p^{n}(t) \ln \left(p^{n}(t)\right)
\end{gathered}
$$

where:

$$
p^{n}(t)=a^{n}(t) a^{-n}(t) / E(t),
$$

to be used to follow the temporal dynamics of the coherent structures of the flow. A small value of $S(t)$ indicates that the corresponding energy is contained in few modes, while a large value of $S(t)$ indicates that the energy is distributed over many modes. A more detailed description of the POD algorithm that has been developed and previous results can be found in Alfonsi \& Primavera [13] and Alfonsi et al. [14].

\section{Results}

$1,820,448$ eigenfunctions $\left(3 \times N_{x} \times N_{y} \times N_{z}\right)$ and correspondent eigenvalues are determined as a result of the decomposition. Figure 1 shows the fluctuating energy content $E(t)$ (9) of the first 5,188 eigenfunctions of the decomposition with time $\left(0 \leq t^{+} \leq 1800\right)$, that alone incorporate $95 \%$ of the total energy content. The plot of $E(t)$ shows a sharp peak at $t^{+}=414$, besides some other smaller spikes. The peak of 
$E(t)$ reflects a sharp rise and subsequent fall of the turbulent activity in the interval $129.6 \leq t^{+} \leq 615.6$ and reveals the occurrence of a turbulent event. The correspondent plot of $S(t)$ (not shown here) shows a sharp fall and rise during the event in a time frame practically concident with that of $E(t)$. The minimum value of $S(t)$ occurs at $t^{+}=284.4$ indicating that during the growing-energy/falling entropy part of the event, the energy arrives to be distributed over a rather low number of flow modes. After $t^{+}=284.4$ the entropy starts growing while the energy is still rising until $t^{+}=414$, the instant corresponding to the peak of the energy within the event cycle, actually followed by another smaller peak at $t^{+}=489.6$. This indicates that for a short time interval $\Delta t^{+}=75.6$ the energy remains high and then rapidly decreases in the interval $489.6 \leq t^{+} \leq 615.6$, redistributing over many modes (the entropy is still rising). The event cycle actually ends at $t^{+}=615.6$. The turbulent event in the whole lasts for $\Delta t^{+}=486\left(129.6 \leq t^{+} \leq 615.6\right), 2.7$ of $10 \delta / u_{\tau}$ time units computed.

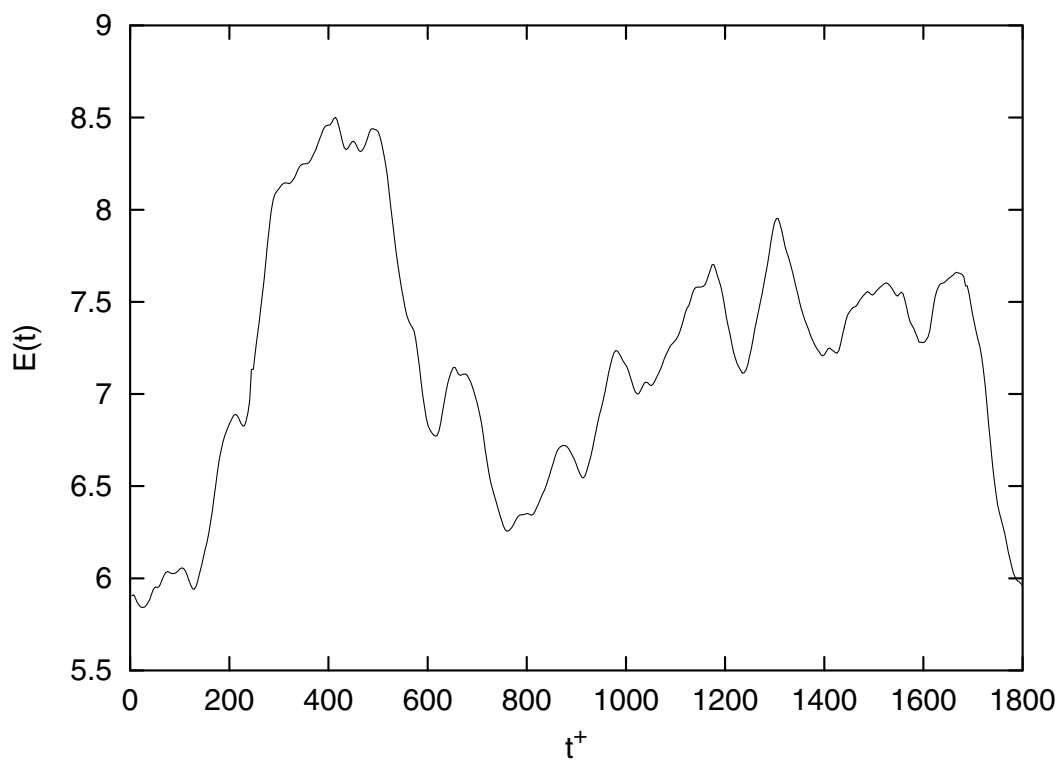

Fig. 1. Fluctuating energy content $E(t)$ (9) (first 5,188 eigenfunctions) with time

Figure 2 shows a visualization of the flow field generated by the first 5,188 eigenfunctions of the decomposition, in terms of isosurfaces of streamwise vorticity. The isovorticity surfaces are represented at $t^{+}=414$, when the energy reaches its maximum. A remarkably large number of flow structures is visible in the wall region of the flow. 


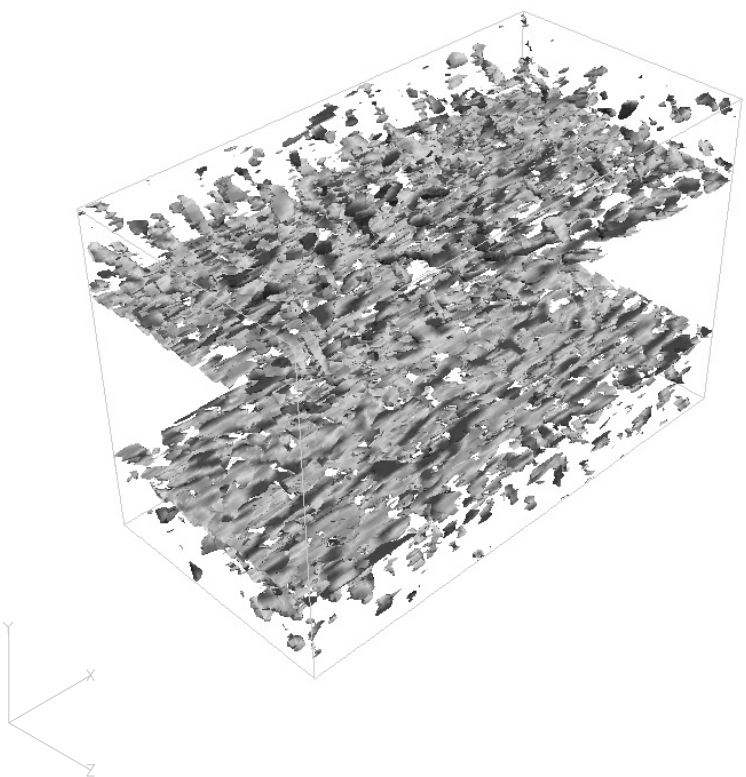

Fig. 2. Isosurfaces of streamwise vorticity at $t^{+}=414$ (first 5,188 eigenfunctions)

\section{Concluding Remarks}

The analysis of the flow field of a numerically simulated turbulent channel flow is performed in terms of flow modes determined with the POD technique. A peak of the kinetic energy of the velocity fluctuations reflects a sharp rise and subsequent fall of the turbulent activity in the interval $129.6 \leq t^{+} \leq 615.6$ and reveals the occurrence of a turbulent event.

\section{References}

1. Robinson S.K.: Coherent motions in the turbulent boundary layer. Ann. Rev. Fluid Mech. 23 (1991) 601

2. Panton R.L.: Overview of the self-sustaining mechanisms of wall turbulence. Prog. Aero. Sci. 37 (2001) 341

3. Kim J., Moin P. \& Moser R.: Turbulence statistics in fully developed channel flow at low Reynolds number. J. Fluid Mech. 177 (1987) 133

4. Moser R.D., Kim J. \& Mansour N.N.: Direct numerical simulation of turbulent channel flow up to $\mathrm{Re}=590$. Phys. Fluids 11 (1999) 943

5. Alfonsi G., Passoni G., Pancaldo L. \& Zampaglione D.: A spectral-finite difference solution of the Navier-Stokes equations in three dimensions. Int. J. Num. Meth. Fluids 28 (1998) 129

6. Passoni G., Alfonsi G., Tula G. \& Cardu U.: A wavenumber parallel computational code for the numerical integration of the Navier-Stokes equations. Parall. Comp. 25 (1999) 593 
7. Passoni G., Cremonesi P. \& Alfonsi G.: Analysis and implementation of a parallelization strategy on a Navier-Stokes solver for shear flow simulations. Parall. Comp. 27 (2001) 1665

8. Passoni G., Alfonsi G. \& Galbiati M.: Analysis of hybrid algorithms for the Navier-Stokes equations with respect to hydrodynamic stability theory. Int. J. Num. Meth. Fluids $\mathbf{3 8}$ (2002) 1069

9. Dean R.B.: Reynolds number dependence of skin friction and other bulk flow variables in two-dimensional rectangular duct flow. J. Fluids Eng. 100 (1978) 215

10. Berkooz G., Holmes P. \& Lumley J.L.: The Proper Orthogonal Decomposition in the analysis of turbulent flows. Ann. Rev. Fluid Mech. 25 (1993) 539

11. Sirovich L.: Turbulence and the dynamics of coherent structures. Part I: coherent structures. Part II: symmetries and transformations. Part III: dynamics and scaling. Quart. Appl. Math. 45 (1987) 561

12. Webber G.A., Handler R.A. \& Sirovich L.: The Karhunen-Loéve decomposition of minimal channel flow. Phys. Fluids 9 (1997) 1054

13. Alfonsi G. \& Primavera L.: Coherent structure dynamics in turbulent channel flow. $J$. Flow Visual. Imag. Proc. 9 (2002) 89

14. Alfonsi G., Restano C. \& Primavera L.: Coherent structures of the flow around a surfacemounted cubic obstacle in turbulent channel flow. J. Wind Eng. Ind. Aerodyn. 91 (2003) 495 